\title{
Photochemistry of interstellar/circumstellar ices as a contributor to the complex organics in meteorites
}

\author{
Michel Nuevo and Scott A. Sandford \\ NASA Ames Research Center, Space Science Division \\ Mail Stop 245-6, Moffett Field, CA 94035, USA \\ email: michel.nuevo-1@nasa.gov, scott.a.sandford@nasa.gov
}

\begin{abstract}
The UV irradiation of interstellar/circumstellar ice analogs is known to lead to the formation of organic compounds such as amino acids and maybe nucleobases. In this work, the mechanisms of formation and distribution of amino acids, chosen as tracers for the organic compounds formed in such experiments, are studied and compared with meteoritic data.
\end{abstract}

Keywords. Astrochemistry, methods: laboratory, ISM: molecules, ultraviolet: ISM, meteorites

\section{Introduction}

Organic molecules have been shown to be formed in interstellar/circumstellar environments consisting of gas and dust particles covered with a thin ice layer containing mainly $\mathrm{H}_{2} \mathrm{O}$, and then $\mathrm{CO}, \mathrm{CO}_{2}, \mathrm{CH}_{3} \mathrm{OH}, \mathrm{NH}_{3}$, and $\mathrm{CH}_{4}$ (Gibb et al. 2004), and subjected to UV-photon and cosmic-ray radiations (Mathis et al. 1983). Those organics are then incorporated into protostellar nebulae, such as the one in which our Solar System formed, as well as into comets and interplanatery dust particles, that can be collected in Earth's atmosphere (Matrajt et al. 2005) and onboard spacecraft (Sandford et al. 2006). Their recent analysis shows the presence of a non-negligible organic fraction. Some compounds, such as amino acids and nucleobases, are of particular interest for prebiotic chemistry. Amino acids have been identified in organic residues formed from the UV irradiation of interstellar ice analog mixtures (Bernstein et al. 2002, Muñoz Caro et al. 2002, Nuevo et al. 2008), and have been detected in carbonaceous chondrites such as Murchison (Cronin \& Pizzarello 1997). However, their detection in the interstellar medium (ISM) is still debated (Kuan et al. 2003). Nucleobases have also been found in meteorites (van der Velden \& Schwartz 1977, Stoks \& Schwartz 1979), but have so far never been observed in the ISM (Charnley et al. 2005).

\section{Experimental setup and protocol}

In a vacuum chamber (a few $10^{-8}$ torr), gases are deposited on a substrate cooled down to $15-20 \mathrm{~K}$. These gases are chosen to mimic the composition of the ISM. Under such physical conditions, the gases condense on the substrate in a thin ice layer, which is simultaneously irradiated by a UV $\mathrm{H}_{2}$ lamp, emitting UV photons at $121.6 \mathrm{~nm}$ (Lyman $\alpha$ ) and around $160 \mathrm{~nm}$. After irradiation, the system is warmed to room temperature, where an organic residue remains on the substrate and can be analyzed. Such residues contain a large variety of compounds, including amino acids (Bernstein et al. 2002, Muñoz Caro et al. 2002, Nuevo et al. 2008). Liquid (LC) and gas (GC) chromatographies are the main techniques used to analyze residues. Such techniques separate residues into their individual compounds by differences of diffusion rates through a chemically-active column. 


\section{Results}

Formation and distribution of amino acids. Analyses of these organic residues show that amino acids always form as long as the starting ice mixture contains the 4 elements C, H, N, and O (Nuevo et al. 2008). The irradiation time and the temperature (10-80 K) have no significant effect on their final distribution. In most of cases, glycine is found to be the most abundant amino acid formed, the abundance of the other compounds decreasing exponentially with their molecular mass, indicating that such compounds were formed via non-biological processes. However, the formation mechanisms of amino acids are not clearly understood. Laboratory studies show that there are in fact several competing mechanisms leading to their formation, and that their distribution in the laboratory is different from what is observed in meteorites (Elsila et al. 2007, Nuevo et al. 2008).

Comparison with meteoritic amino acids. The abundances of the 3 most abundant amino acids formed in laboratory organic residues, namely glycine, alanine, and serine, have been compared with their abundances in $1 \mathrm{~g}$ of the Murchison meteorite (Nuevo et al. 2008 and references therein). In the residues, alanine was found to be produced with abundances half that of glycine, whereas in Murchison these two amino acids have similar abundances. Serine is formed with lower relative abundances in Murchison than in residues. These discrepancies indicate that the formation mechanisms of amino acids are different in the laboratory and in meteorites, or that Murchison had subsequent alteration, such as the (photo-) chemical process or decomposition of high molecular mass amino acids into smaller compounds such as glycine and alanine. However, this comparison is biased since mainly only proteinaceous amino acids have been searched for in organic residues (Nuevo et al. 2008). The search for non-proteinaceous amino acids such as $\alpha$-aminobutyric acid or isovaline in residues, which have been detected in Murchison, may allow a better understanding of the formation mechanisms of such compounds.

Nucleobases. Nucleobases are complex molecules consisting of one (pyrimidine-based) or two (purine-based) rings containing nitrogen atoms. They are probably key compounds for prebiotic chemistry. The formation and photo-stability of pyrimidine- and purinebased compounds are currently being studied at NASA Ames, where pyrimidine mixed with $\mathrm{H}_{2} \mathrm{O}$ ice is UV irradiated at low temperature. The early results show that such mixtures lead to the formation of nucleobase-like compounds.

\section{References}

Bernstein, M., Dworkin, J., Sandford, S., Cooper, G., \& Allamandola, L. 2002, Nature, 416, 401 Charnley, S. B., et al. 2005, Adv. Sp. Res., 36, 137

Cronin, J. R. \& Pizzarello, S. 1997, Science, 275, 951

Elsila, J. E., Dworkin, J. P., Bernstein, M. P., Martin, M. P., \& Sandford, S. A. 2007, ApJ, 660, 911

Gibb, E. L., Whittet, D. C. B., Boogert, A. C. A., \& Tielens, A. G. G. M. 2004, ApJS, 151, 35

Kuan, Y.-J., Charnley, S. B., Huang, H.-C., Tseng, W.-L., \& Kisiel, Z. 2003, ApJ, 593, 848

Mathis, J. S., Mezger, P. G., \& Panagia, N. 1983, A\&A, 128, 212

Matrajt, G., Muñoz Caro, G. M., Dartois, E., d'Hendecourt, L., Deboffle, D., \& Borg, J. 2005, $A \mathscr{S} A, 433,979$

Muñoz Caro, G. M., Meierhenrich, U. J., Schutte, W. A., Barbier, B., Arcones Segovia, A., Rosenbauer, H., Thiemann, W. H.-P., Brack, A., \& Greenberg, J. M. 2002, Nature, 416, 403

Nuevo, M., Auger, G., Blanot, D., \& d'Hendecourt, L. 2008, Orig. Life Evol. Biosph., 38, 37

Sandford, S. A., et al. 2006, Science, 314, 1720

Stoks, P. \& Schwartz, A. 1979, Nature, 282, 709

van der Velden, W. \& Schwartz, A. 1977, Geochim. Cosmochim. Acta, 41, 961 